\title{
Percepción del profesorado respecto a los efectos de la separación y divorcio
}

\section{Teacher perceptions about the effects of separation and divorce}

\author{
Beatriz Alonso, Andrea Montes, Francisca Fariña y Mª José Vázquez
}

Universidad de Vigo

\begin{abstract}
Resumen
El objetivo de este trabajo consiste en analizar la opinión del profesorado de Primaria sobre los efectos que tiene en el alumnado la separación/divorcio de sus progenitores. Para ello se aplicó un cuestionario a 146 maestros/as en activo. Los resultados señalan que el profesorado advierte que la ruptura parental repercute en todos los ámbitos en los que se desenvuelve el menor. Además, consideran que la formación universitaria es insuficiente para tratar esta problemática; tampoco han participado en programas de intervención y desconocen los existentes en Galicia. En conclusión, perciben las necesidades de estas familias y la necesaria formación para afrontarlas. Palabras clave: separación, divorcio, profesorado, conflicto, menores.
\end{abstract}

\begin{abstract}
The objective of this paper is to analyze the opinion of Primary teachers about the effects on students separation / divorce of their parents. A questionnaire was applied to 146 teachers in active. The results show that teachers warns that parental break affects all areas in which the child develops. They also consider that university education is insufficient to address this problem; not have they participated in intervention programs and ignore existing in Galicia. In conclusion, they perceived needs of these families and the necessary training to deal with them.
\end{abstract}

Keywords: separation, divorce, teachers, conflict, children.

\section{Método}

\section{Participantes}

Para este estudio contamos con 146 maestros/as que trabajan en Educación Primaria, de los cuales 29 son hombres y 116 mujeres. La edad media se sitúa en 43.38, siendo la edad mínima 22 y la máxima 61 años. En cuanto a su formación, el $71.2 \%$ son diplomados, el $12.3 \%$ diplomados y licenciados, el $10.3 \%$ licenciados, el $2.7 \%$ son graduados y el $1.4 \%$ son licenciados y graduados. Estos disponen de experiencia, siendo la media de años trabajados 18.54, con un máximo de experiencia docente de 38 años. En cuanto a las características del centro de trabajo, están ubicados en Pontevedra, de los cuales el $71.9 \%$ pertenecen al ámbito urbano y el $28.1 \%$ al rural. De éstos, el $66.4 \%$ son públicos y el $33.6 \%$ privados-concertados.

\section{Procedimiento e instrumento}

Se aplicó el cuestionario "Percepción del profesorado en procesos de separación y divorcio" adaptándolo a los participantes (véase, Anexo I). Esta prueba fue creada ad hoc por el grupo de investigación PS1 (Fariña et al., en prensa). Este cuestionario ha sido complementado de manera individual. De forma previa, se explica a la población participante el objeto del estudio y la finalidad del mismo. Del mismo modo, se ha indicado que la participación es voluntaria y que los datos se van a utilizar única y exclusivamente con fines científicos, preservando el anonimato de los mismos, según la Ley Orgánica 15/1999, de 13 de diciembre, de Protección de Datos de Carácter Personal. Consta de 24 ítems con una escala de respuesta, tipo Likert, con cinco niveles (1 que indica muy en desacuerdo a 5 que indica muy de acuerdo). Este instrumento aborda tópicos tales como:

1) Grado de sensibilización y actitud sobre los procesos de separación y divorcio. Alude a cuestiones que hacen referencia a la valoración afectiva y cognitiva del impacto de los procesos de separación y divorcio en los estudiantes de la etapa educativa en la que imparten docencia.

2) Grado de conocimiento sobre la incidencia de los procesos de separación y divorcio en sus estudiantes. Introduce cuestiones que recogen la percepción que tiene el profesorado acerca del nivel de conocimiento y habilidades que tiene con relación a la problemática objeto de estudio.

3) Grado de experiencia sobre la intervención que deberían tener los procesos de separación y divorcio en el ámbito escolar. Se recoge el nivel de experiencia profesional que tiene/ha tenido el profesorado con relación al afrontamiento y gestión de los procesos de separación y divorcio que están o han experimentado sus alumnos/as.

\section{Resultados}

El profesorado de Educación Primaria que ha colaborado en este estudio considera que la separación/divorcio de los progenitores del alumno/a puede tener repercusiones dentro y fuera del ámbito escolar, por lo tanto los centros educativos pueden verse inmersos en los conflictos de las rupturas de pareja. De este modo, el profesorado puede ayudar a los/as alumnos a afrontar la separación de sus padres, aunque consideran complicado paliar los efectos negativos producidos por 
este evento. Así, cabe destacar que es responsabilidad de todos, no solo del docente, ayudar a prevenirlos.

Por otro lado, estos profesionales creen que han recibido formación sobre la problemática de la separación/divorcio, pero consideran que ésta no es suficiente, por lo que en la enseñanza universitaria debería dedicarse más tiempo a enseñar cómo intervenir, ya que existen dudas acerca de cómo actuar con el menor en el momento real en el que se produce el conflicto.

El profesorado encuestado observa que los alumnos/as cuyos progenitores se han separado/divorciado presentan mayor riesgo de comportamientos disruptivos y antisociales, así como cambios en la motivación hacia los estudios y en las expectativas de futuro. Además esta circunstancia, generalmente, conlleva para la familia problemas judiciales, económicos y relacionales.

En este sentido, este sector profesional muestra preocupación ante un alumno/a que está sufriendo con la separación/divorcio de sus progenitores, tanto es así que opinan que los/as alumnos/as que viven en familias intactas, con alto nivel de conflicto entre los padres, se encuentran mejor que aquellos cuyos progenitores están separados/divorciados pero que mantienen una buena relación.

Además, según la percepción de este profesorado, el acontecimiento objeto de estudio se incrementa cada año, por lo que la comunicación con las familias se vuelve fundamental, pero ésta resulta más compleja cuando existe un alto nivel de conflicto y baja colaboración entre los progenitores. Asimismo creen que los recursos con los que cuentan los centros de enseñanza son suficientes para intervenir con estos menores.

En cuanto a la experiencia profesional docente, estos profesores han tenido que afrontar y gestionar algún caso de un alumno/a cuyos padres se habían separado/divorciado, asimismo han participado en programas de intervención, puesto que conocen la existencia de éstos en nuestra comunidad autónoma. Debido a esta experiencia, son conscientes de que cuando los padres se separan/divorcian, el centro educativo debe de informar únicamente al progenitor custodio de la evolución escolar del alumno/a (véase Tabla 1).

Tabla 1.

Percepción del profesorado acerca de los efectos de la separación y el divorcio en sus alumnos/as .

\begin{tabular}{|c|c|c|c|}
\hline Ítems & $\mathrm{T}$ & M & Dif-M \\
\hline Consecuencias en el ámbito escolar & $26.020 *$ & 4.48 & 1.479 \\
\hline $\begin{array}{l}\text { El profesorado puede ayudar a } \\
\text { afrontar la separación }\end{array}$ & $10.009 *$ & 3.69 & .692 \\
\hline $\begin{array}{l}\text { Los centros pueden verse inmersos en } \\
\text { los conflictos }\end{array}$ & $17.324 *$ & 4.23 & 1.234 \\
\hline Dudas sobre cómo intervenir & $2.651 * *$ & 3.25 & .247 \\
\hline $\begin{array}{l}\text { Incremento del alumnado de } \\
\text { progenitores separados }\end{array}$ & $12.725^{*}$ & 3.96 & .957 \\
\hline $\begin{array}{l}\text { Preocupación ante un alumno inmerso } \\
\text { en este proceso }\end{array}$ & $31.370 *$ & 4.62 & 1.615 \\
\hline $\begin{array}{l}\text { La prevención de los efectos negativos } \\
\text { es responsabilidad de todos }\end{array}$ & $7.916^{*}$ & 3.72 & .717 \\
\hline $\begin{array}{l}\text { El profesorado no puede hacer nada } \\
\text { para paliar los efectos negativos }\end{array}$ & $-12.008 *$ & 2.09 & -.911 \\
\hline
\end{tabular}

Formación universitaria del profesorado es suficiente

Existencia de programas de intervención en Galicia

Recursos de los centros suficientes

La separación/divorcio puede afectar negativamente a los hijos/as.

La separación/divorcio puede afectar negativamente en el rendimiento académico

Mayor riesgo de comportamientos disruptivos

Cambios en la motivación hacia los estudios y expectativas de futuro

Problemas judiciales, económicos y relacionales para las familias

Alumnado de familias intactas con alto nivel de conflicto se encuentran mejor que los separados/divorciados con buena relación

Comunicación familia-escuela más compleja con alto nivel de conflicto

El centro debe informar solo al progenitor custodio

He gestionado un caso de un alumno cuyos padres se han separado

He participado en programas de intervención

Considero que se debería adquirir formación específica sobre separación/divorcio

Nota. ${ }^{*} \mathrm{p}<.001 ; * * \mathrm{p}<.01 ; * * * \mathrm{p}<.05$

\section{Discusión}

Las parejas o los matrimonios que han atravesado una crisis y no fueron capaces de superarla, acaban tomando la decisión de separarse o divorciarse. Los datos del Instituto Nacional de Estadística (INE) evidencian que cada vez son más las parejas que optan por esta solución. De hecho un informe de octubre del año 2014 sobre la ruptura conyugal en España en el año 2013 indica que durante ese año se produjeron un total de 100.437 sentencias de nulidades, separaciones y divorcios, lo que supuso una tasa de 2.1 por cada 1.000 habitantes. Analizando estos datos y comparándolos entre sí, se observa un incremento en el número de rupturas del $0.8 \%$ en el año 2013, con respecto al 2012.Según el tipo de resolución de conflictos, los divorcios representaron el $95 \%$ del total, las separaciones el $4.9 \%$ y las nulidades el $0.1 \%$ restante. Del total de divorcios, el $75.4 \%$ se resolvieron de mutuo acuerdo frente al $24.6 \%$ por la vía contenciosa. Por otra parte, las separaciones de mutuo acuerdo representaron el $83.9 \%$ y las contenciosas el $16.1 \%$ (véase Figura 1). 


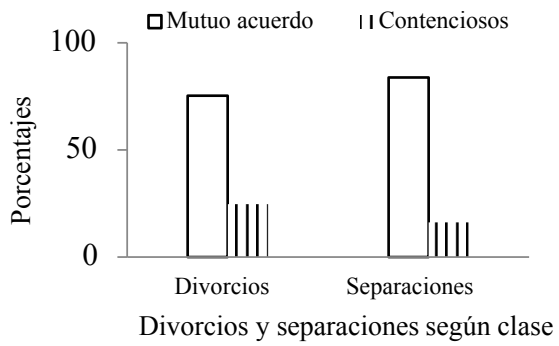

Figura 1. Estadísticos separación y divorcio año 2014.

Con relación a este proceso han surgido múltiples estudios centrados en analizar la incidencia e impacto que esta experiencia tiene para los miembros de la familia, así, Despert (1965) afirma que lo patológico no es el proceso de separación y divorcio, sino la forma de afrontar este proceso. En este caso, el conflicto recurrente y el consiguiente estrés es lo que resulta nocivo para quienes tienen que superarlo $\mathrm{y}$, en consecuencia, aceptarlo y superarlo. Según Amato (2010), la vivencia dentro de un matrimonio disfuncional origina un estrés significativo que puede acabar produciendo ansiedad y depresión. Así pues, la gestión que se haga de este proceso va a incidir en el impacto que éste tenga en los hijos menores; en este sentido, los estudios advierten que los efectos del divorcio en los niños y las niñas están íntimamente relacionados con el ajuste emocional de los progenitores $y$, muy especialmente, con la relación postdivorcio que éstos mantengan (Fariña, Seijo, Novo y Arce, 2014). Por tanto, el clima de tensiones, batallas y discordias constantes en el hogar junto con la actitud negativa de los padres y las madres tiene peores repercusiones para los hijos y las hijas que el trauma de la ruptura. De hecho, los niños y las niñas de familias intactas, con alto nivel de conflicto, obtienen peores resultados en valoraciones psicológicas que los de familias divorciadas con bajo nivel de conflicto (Amato, 1991).

Por otra parte, el impacto del divorcio y la separación en los menores dependen de su nivel de desarrollo. Concretamente, los y las preescolares tienden a manifestar conductas regresivas: insomnio, crisis de rabietas, angustia de separación, pérdida del control de esfínteres, regresión en los hábitos de limpieza, estancamiento en las adquisiciones cognitivas, temores fóbicos y sentimientos de culpabilidad. Además, muestran su ira intensa contra uno o ambos padres al mismo tiempo y pueden desarrollar cuadros depresivos, lo que conlleva una disminución del rendimiento académico y un deterioro en las relaciones con sus compañeros y compañeras (Wallerstein, 1991).

Por ello, es muy importante estudiar este tema, ya que, en muchas ocasiones, se desconocen las posibles repercusiones que tiene este evento en los niños/as y cómo puede repercutir en el ámbito escolar. En este sentido, la literatura significa la necesidad de conocimiento tanto en los padres como en los docentes, de manera que se puedan detectar cambios derivados de esta situación para poder prevenirlos y tratarlos.
En este sentido, los datos resultantes del presente estudio señalan que el profesorado de Educación Primaria considera que la separación y el divorcio de los progenitores tiene una repercusión negativa en los alumnos/as, incidiendo negativamente en su aprendizaje. Además, aprecian que puede influir tanto en su motivación académica como en sus expectativas de futuro. Asimismo, refieren que estos alumnos presentan riesgo de emitir comportamientos disruptivos. Por otra parte, informan que la comunicación entre familia y profesorado resulta más compleja cuando existe un alto grado de conflicto y baja colaboración entre los progenitores.

En línea con lo que establece la literatura sobre el tema, los/as maestros/as de Primaria constatan que la separación y el divorcio inciden de forma desfavorable en la vida escolar de los/as menores, así, se estima que es fundamental la experiencia del maestro o la maestra a la hora de saber detectar los desajustes que se pueden producir en el ámbito escolar tras la separación o el divorcio (Castells, 2004; Musitu, 2004; Storksen, Thorsen, Overland y Brown, 2012). En esta línea, según Velasco-Pereira, Vázquez y Fariña (2014), el tipo de estructura familiar influye de forma significativa en la variable académica repetir curso, pues los estudiantes que experimentan la separación de sus progenitores repiten más cursos, lo que se puede entender como evidencia de vulnerabilidad a presentar dificultades e incluso fracaso escolar. De este modo, la experiencia del profesorado es un factor clave para detectar tal debilidad y poder intervenir con el fin de solventar la situación.

De esta forma, desde edades tempranas la separación y el divorcio de los padres puede suponer una vivencia negativa para los/as hijos/as. En este contexto se enmarca la mediación familiar, un medio de solución de conflictos que permite a las partes gestionarlos positivamente, encontrando soluciones efectivas y que posibilite la adquisición de habilidades para evitarlos (Fariña y Pillado, 2015).

Comentado lo anterior, podemos afirmar que, en general, los análisis de los resultados anteriormente obtenidos apoyan que la ruptura de los progenitores puede estar asociada con una serie de consecuencias adversas en la vida de los hijos, tal y como manifiesta la literatura (Fariña, Arce, Novo, Seijo, 2013) y más concretamente, con la afectación del área escolar y académica.

\section{Referencias}

Amato, P. R. y Keith, B. (1991). Parental divorce and adult well-being: A meta-analysis. Journal of Marriage and the Family, 53, 43-58.

Amato, P. (2010). Research on Divorce: Continuing Trends and New Developments. Journal of Marriage and Family, 72, 650-666.

Despert, J.L. (1965). The emotionally disturbed child. New York: Brunner.

Fariña, F., Arce, R., Novo, M., y Seijo, D. (2013). De las necesidades de los menores a la intervención: un 
programa con menores en riesgo de desestructuración familiar. En M. Isorna (Coord.), Prevención de drogodependencias $y$ otras conductas adictivas. Madrid: Pirámide.

Fariña, F., Seijo, D., Novo, M., Arce, R. (2014). La justicia terapéutica en procesos de ruptura de pareja: El papel del psicólogo. En C. Chan, C. Estrada, y F.J. Rodríguez (Eds.), Aportaciones a la psicología jurídica y forense desde Iberoamérica (pp. 113-134). México, DF: Manual Moderno.

Fariña, F. y Pillado, E. (2015). Mediación familiar. Editorial Tirant Lo Blanch.

Instituto Nacional de Estadística. (2014). Consultado el 11 de noviembre de 2014 en http://www.ine.es/prensa/np867.pdf

Ley Orgánica 15/1999, de 13 de diciembre, de Protección de Datos de Carácter Personal (1999). Jefatura del Estado. España.
Musitu (2004). Introducción a la psicología comunitaria. Barcelona: UOC.

Storksen, I., Thorsen, A. A., Overland, K., y Brown, S. R. (2012). Experiences of daycare children of divorce. Early Child Development and Care, 182 (7), 807-825. doi:http://dx.doi.org/10.1080/03004430.2011.585238

Velasco-Pereira, P., Vázquez, Mª J. y Fariña, F. (2014). Influencia de la estructura familiar en la superación del curso académico de los hijos: familias padres separados VS familias intactas. VIII Congreso (Inter)Nacional de Psicología Jurídica y Forense.

Wallerstein, J. (1991). Children of divorce: Preliminary report of a ten-year follow-up of older children and adolescents. Journal of the American Academy of Child and Adolescent Psychiatry, 30, 349-360. 